\title{
The CD38/CD157 mammalian gene family: An evolutionary paradigm for other leukocyte surface enzymes
}

\author{
Silvia Deaglio • Fabio Malavasi
}

Received: 9 December 2005 / Accepted: 12 January 2006/ Published online: 30 May 2006

(C) Springer Science + Business Media B.V. 2006

\begin{abstract}
Human CD38 is the mammalian prototype of a family of phylogenetically conserved proteins which share structural similarities and enzymatic activities involved in the production of an intracellular second messenger with calcium mobilizing effects. Engagement of CD38 by agonistic monoclonal antibodies and the CD31 ligand initiates a cytoplasmic signaling cascade involving tyrosine phosphorylation of the proto-oncogene c-cbl and of the extracellular regulated kinase 1 of 2 complex. Further requirements for signal transduction include a privileged localization within the cholesterol-rich areas of the plasma membrane and physical association with specialized surface receptors. CD38-mediated signals are crucial in heterotypic cell adhesion and migration as well as in the activation of proliferation/survival programs by normal and neoplastic cells. Here we review the most recent literature on this complex topic and attempt to formulate a single model reconciling the enzymatic and receptorial activities of CD38.
\end{abstract}

Keywords calcium mobilization · cell surface enzymes · cell surface receptors $\cdot$ intracellular signaling
Abbreviations
BM bone marrow
cADPR cyclic ADP ribose
IP3 inositol triphosphate

S. Deaglio · F. Malavasi $(\bullet)$

Laboratory of Immunogenetics, Department of Genetics,

Biology and Biochemistry, and Research Center

on Experimental Medicine,

University of Torino Medical School,

via Santena, 19, 10126 Torino, Italy

e-mail: fabio.malavasi@unito.it
ERK1/2 extracellular regulated kinase 1/2

mAbs monoclonal antibodies

NAD nicotinamide adenine dinucleotide

\section{Introduction}

The concept of cell surface molecules with a catalytically active site in the extracellular environment was lastly brilliantly analyzed in a dedicated issue of Immunological Reviews edited by Goding and Howard [1]. At the time it was becoming clear that ectoenzymes could no longer be considered an oddity in leukocyte biology; on the contrary, it was seen that $\sim 3.5 \%$ of the molecules expressed on the surface of human and murine cells shared such characteristics. This finding galvanized the attention and interest of basic scientists and, later, of clinical scientists as well.

Nucleotide-metabolizing ectoenzymes constitute a family within this larger family and are represented by a set of molecules involved in the catabolism and scavenging of extracellular nucleotides. This process results in the synthesis of compounds that play a critical role in cell homeostasis and metabolism, suggesting that the physiological role of this complex family goes beyond the simple recycling of nucleotides.

One of the characteristics initially thought to be shared by several, even though not all, nucleotidemetabolizing ectoenzymes was the ability to work in an environment where the substrate was generally present only in trace amounts and where the final product would be used prevalently inside the cell [2]. This initial view of enzymes working in an antieconomical environment was later refined thanks also 
to the more sophisticated experimental approaches confirming that substrates and final products are not so topologically confined [3].

The majority of these molecules, identified using the grid of the CD International Workshop on Differentiation Antigens and considered to be orphan molecules, found initial clinical applications as disease markers. Indeed, CD38 is still considered an 'activation marker,' useful in the diagnosis of leukemias and myelomas [4], while CD39 was initially defined as a marker of EBV infection [5]. The same fate was shared by CD26 [6] and CD10/CALLA [7], probably the most commonly used clinical markers, as well as PC-1 [8], one of the first reliable markers of human plasma cells.

This review will focus on the CD38/CD157 family of ADP ribosyl cyclases and their relevance in physiology and human pathology.

\section{CD38 and CD157: The early days}

CD38 was identified during detailed analysis of the cell surface by means of monoclonal antibodies (mAbs) as carried out by EL Reinherz and SF Schlossman during their pioneer search for molecules acting as T-cell receptors and as transducers of the signals elicited by the encounter with the specific antigen [9]. The upshot of that approach was the definition of a vast array of molecules, including some involved in the economy of discrete subsets of cells (CD4 and CD8), some playing the more general role of metabolic regulators (CD71/ TFR-1) and several others, such as CD38, that served as markers for the study of thymocytes, activated $\mathrm{T}$ cells and selected tissues [10,11].

Our interest in investigating human CD38 was sparked by the distribution of the molecule, which ranged from discrete expression during thymic differentiation to an extremely limited presence during the normal physiological life of a $\mathrm{T}$ cell. The molecule was re-expressed at high density by $\mathrm{T}$ cells undergoing activation and in selected leukemias [12]. In apparent contrast to the simplistic view of CD38 an activation marker was the finding that terminally differentiated plasma cells and their pathological counter-parts expressed the highest surface amount among human cells.

Our analysis proceeded from the observation that $\mathrm{CD} 38$, a $45 \mathrm{kD}$ molecule, was initially reported as being associated to a $12 \mathrm{kD}$ single chain protein, reminiscent of a family of HLA Class I-like molecules known at that time as T6 (now CD1a) [13]. That view was disconfirmed once we generated an extensive panel of mAbs specific for the molecule, which enabled us to perform an initial structural analysis that ruled out the presence of $\beta 2$-microglobulin. Moreover, using a somatic cell genetics approach, the gene coding for the structure was attributed to human chromosome 4 ( $4 \mathrm{p} 15$, and thus outside the canonical HLA supergene in chromosome 6) [14].

One of the mAbs in the panel used for the characterization of human CD38 was found to induce signals after binding to its target, a new and unforeseen characteristic of mAbs [15]. An agonistic mAb is usually a reagent with a very high affinity for the target, mimicking the interaction between ligand and receptor. Using that agonistic $\mathrm{mAb}, \mathrm{CD} 38$ was initially reported as a surface molecule acting as a receptor engagable by an unknown ligand [16]. While we were busy analyzing the molecule from an immunological perspective, CD38 unexpectedly became the focus of a group of biochemists who were interested in the regulation of calcium signaling through a pathway independent from inositol triphosphate (IP3) [17]. H.C. Lee was able to identify cyclic ADP ribose (cAPDR) as an intracellular second messenger and to purify the enzyme responsible for its synthesis from Aplysia californica, a mollusk separated from humans by 80 million years. Surprisingly, the sequence of soluble ADP ribosyl cyclase purified from Aplysia proved strikingly similar to the leukocyte transmembrane antigen CD38.

The similarity in amino acid sequences between Aplysia ADP ribosyl cyclase and the human lymphocyte antigen CD38 was timidly reported as a letter which appeared in TIBS in December 1992 [18]. This led to an explosion in interest by basic and clinical scientists, astonished by the close similarity between two such phylogenetically distant molecules. Human and mouse CD38 were confirmed to be ectoenzymes $[19,20]$. The underlying issue that had to be resolved concerned the relationship between the molecule's receptorial and enzymatic activities: In other words, are the signals implemented by CD38 ligation guided by the intermediate or final products synthesized by CD38, or are the two functions independent?

The identification of a second member of the CD38 family occurred during roughly the same years [21]. Bone marrow stromal antigen-1 (BST-1), later clustered as CD157, was defined as a stromal cell molecule facilitating pre-B cell growth [22]. Its deduced amino acid sequence exhibited $33 \%$ sequence homology with CD38. Originally, BST-1/CD157 gene was mapped to chromosome 14q32.3, where immunoglobulin heavy 
chain genes are clustered [22]. Further studies did not confirm that initial finding and instead mapped CD157 to chromosome $4 \mathrm{p} 15$, in close proximity to the CD38 gene [23].

This review is an attempt to present a unified picture that encompasses both CD38 and CD157, providing answers to several issues that have remained unresolved after more than a decade of analysis.

\section{CD38 as a signaling molecule}

As mentioned, analysis using agonistic mAbs eventually led to characterization of the signaling pathway controlled by CD38. Because the molecule is widely expressed by lymphoid cells, the signaling events were analyzed in detail in distinct lineages and during discrete developmental stages. The emerging picture is complex and non-univocal, but some common threads seem to emerge.

a) CD38 engagement by agonistic mAbs is followed by a signaling cascade typical of the canonical receptors, including tyrosine phosphorylation of a sequential number of intracellular enzymes, nuclear events and more long-term effects dependent on active protein synthesis. The individual steps of the signaling pathway vary according to the model studied; however, a common player in the CD38 pathway is the proto-oncogene c-cbl, which is reported to be tyrosine phosphorylated in all the cell models tested, including T, B, NK and myeloid cells [24-28]. This observation suggests that CD38 may play a role in the fine tuning of antigen receptor signaling [29]. Moving further down, the signal appears to be funneled through the ERK1/2 cross-point, at least in T and NK cells [28, 30].

b) An increase of the cytoplasmic levels of calcium ions is also a common theme upon activation of CD38. The calcium wave is typically low and slow in rising as compared to the spikes obtained after signaling through the antigen receptors in $\mathrm{T}$ and $\mathrm{B}$ lymphocytes. Unlike the antigen receptors, a CD38-induced calcium wave may last several minutes before declining, indicating that the molecular mechanisms responsible for calcium mobilization might be different [27, 31, 32]. In several instances, it was possible to determine PLC- $\gamma$ tyrosine phosphorylation upon CD38 crosslinking, suggesting that the conventional IP3 pathway may account for calcium mobilization [33, 34]; in other instances and mostly using murine models, no PLC- $\gamma$ activation could be detected [35]. However, a direct contribution of cADPR extracellularly produced by CD38 to the signaling process cannot be convincingly ruled out, as the deletion of the catalytically active site by means of site-directed mutagenesis is followed by a lack of binding by the available agonistic mAbs $[36,37]$. Moreover, activation of surface CD38 by preincubation of cells with nicotinamide adenine dinucleotide (NAD+) was found to lead to a dosedependent increase in cADPR, with no recordable changes in the intracellular concentrations of calcium ions [38, 39].

c) What ignites the signal? The lack of correlation between CD38-mediated signals and the production of cADPR, ADPR, and/or nicotinamide prompted the search for alternative ligands capable of initiating the signaling process. The finding that $\mathrm{CD} 38$ could modulate $\mathrm{CD} 4^{+} / \mathrm{CD} 45 \mathrm{RA}^{+}$naïve $\mathrm{T}$ lymphocyte [40] adhesion to endothelial cells culminated with the identification of a $130 \mathrm{kD}$ protein recognized by a soluble form of CD38 that was used as a probe in a Western blot system [41]. This molecule turned out to be CD31/PECAM-1, an Ig superfamily member mainly involved in the modulation of leukocyte adhesion to the vessels [42]. In subsequent years, most of the signals recorded using agonistic mAbs were reproduced by using the CD31 ligand. These included mobilization of calcium signaling as well as more structured events, such as proliferation and cytokine induction [31, 43]. More recently, a similar approach exploiting a soluble CD38-Ig recombinant protein was used to identify novel ligands in the murine system. As a result, a ligand of molecular weight $130 \mathrm{kD}$, apparently different from CD31, was detected on the surface of dendritic cells [44],

d) The structural requirements for signaling are as yet unclear. Of the various challenges facing defenders of the receptor hypothesis, determining how the signaling is initiated is perhaps the most compelling. The role of the cytoplasmic tail is controversial at this point. On the one hand, the Lund group convincingly showed that the presence of the intracellular residues of CD38 is irrelevant for signals and enzymatic activities [38]. Moreover it is generally accepted that the tail does not contain signaling motifs. However, other studies have determined - at least in $\mathrm{T}$ lymphocytes - a direct association between the tail of CD38 and the SH2 domain of the kinase lck [45]. 
We and others have shown that CD38 is located in critical areas of the plasma membrane in close physical proximity with professional signaling receptors [46], such as the $\mathrm{T}$-cell receptor in $\mathrm{T}$ lymphocytes [34], the B-cell receptor complex in B cells [47], CD16 in NK cells [30], MHC Class II and CD9 in monocytes [27, 48] and the CCR7 chemokine receptor, CD83 and CD11b in mature dendritic cells [49]. Although these associations are clearly visible using confocal microscopy, they are lost when performing co-immunoprecipitation experiments, suggesting either that the interaction is weak or that there is an as yet unidentified third party protein which acts as a bridge between CD38 and the other receptors. In line with the latter hypothesis, recent papers have implied the existence of supramolecular complexes acting in conjunction with multiple partners $[48,50]$. These complexes appear to reside within the cholesterolrich areas of the plasma membrane [51] and to be dependent on their integrity [52]. The association between CD38 and other signaling receptors was deemed to have a functional nature, as implied by the results of experiments with T, B and NK cell line models genetically modified to lack functional signaling receptors [30, 34, 53]. This hypothesis was also confirmed by using normal cells that lack functional receptors [30].

e) The long-term events described as a consequence of the interaction of CD38 with the CD31 ligand and mimicked by agonistic mAbs vary according to cell lineage and differentiation status. A common theme in human $\mathrm{T}$ cells is the modulation of a pattern of cytokines, including the classical Th1 (IL-1, IL-6 and IFN- $\gamma$ ) as well as the Th2 cytokines (IL-4 and IL-10) [54, 55]. The result is implementation of a network of signals, which ultimately rule the processes of proliferation, survival and differentiation. This was recently shown in tests of the receptor hypothesis using chronic lymphocytic leukemia (CLL) as a model [47]. CD38 was found to be expressed by a subset of CLL cells characterized by shorter survival times and shorter intervals prior to therapy [5658]. In these cells, CD38-mediated signals was shown to lead to cell proliferation and to increase the survival of the neoplastic clone by cooperating with other survival receptors, including semaphorin CD100 [43]. The signals induced by the agonistic $\mathrm{mAb}$ are fully mimicked after interaction with the CD31 ligand, suggesting that this receptor/ligand system may play a direct role in the pathogenesis of CLL.

\section{CD38 and CD157 as enzymes}

As mentioned above, the extracellular domains of CD38 and CD157 contain an enzymatic site that can synthesize cADPR and nicotinic acid adenine dinucleotide phosphate (NAADP) from NAD ${ }^{+}$and NADP ${ }^{+}$, respectively [19, 59]. Thus, $\mathrm{CD} 38$ and $\mathrm{CD} 157$ are ADP-ribosyl cyclases, a family of multifunctional enzymes apparently ubiquitous in eukaryotic cells [60]. CD38 and CD157 are the major enzymes with ADP-ribosyl cyclase activity, although a non-CD38/ CD157 ADP-ribosyl cyclase has recently been detected in brain tissue from CD38 knock-out (KO) mice [61]. The most relevant product of the reaction is cADPR, a universal second messenger with the ability to control calcium levels in an IP3 independent way [62]. cADPR - and thus ADP ribosyl cyclases - play a key role in physiological processes, including cell proliferation [63], muscle contraction [64, 65], stem cell regeneration [66] and hormone secretion [67], among the most relevant. Under normal physiologic pH conditions, and unlike the Aplysia enzyme, CD38 is a very inefficient cyclase, with cADPR representing only $1 \%-3 \%$ of the final products [19]. ADPR and NAADP produced by CD38 [68] also cooperate in the regulation and modulation of intracellular calcium: Indeed, ADPR acts on plasma membrane LTRPC2 calcium channels, and NAADP mobilizes calcium from intracellular stores [69]. Basile et al. recently showed that CD38 and the other ADP ribosyl cyclases also synthesize three novel adenine dinucleotides from cADPR [70]. Pinpointing the role of these compounds in intracellular calcium homeostasis is in the early stages: Preliminary data indicate that they may play relevant roles in the network of positive and negative signals interchanged between cells expressing CD38 or CD157 and interacting partners.

The ADP ribosyl cyclase family of enzymes is present in a range of cells ranging from protists and unicellular algae to higher Metazoa and Metaphyta. Even if CD38 and CD157 share low sequence identities $(\sim 30 \%)$, genetic analyses have shown that the exon-intron structures of the two genes is remarkably similar, suggesting that they evolved through gene duplication phenomena from a common ancestor [71]. The similarities are maintained when comparing CD38/CD157 with the Aplysia cyclase [72]. The extracellular domain of CD38 was recently crystallized and compared to the structures of CD157 and of the Aplysia cyclase [73-76]. The results indicate that the overall topology of the three enzymes is similar: However, the large structural changes observed at the two termini of the extracellular domains of CD38 
suggest that this gene underwent heavier evolutionary stress than the CD157 and Aplysia ADP ribosyl cyclase genes.

\section{CD157}

In contrast with CD38, CD157 is linked to the plasma membrane by means of a glycosylphosphatidylinositol (GPI) anchor. Another element distinguishing it from CD38 concerns cell and tissue distribution, with CD157 detected on human peripheral blood monocytes, eosinophils, basophils, neutrophils and on the majority of myeloperoxidase-positive bone marrow (BM) myeloid precursors. Erythrocytes, platelets and lymphoid cells from peripheral blood or from spleen and tonsils, as well as lymphoblastoid cell lines of $\mathrm{T}$ and B origin are negative [77]. Outside the immune system, CD157 is expressed by the endothelium, by synovial cells and derived lines and by follicular dendritic cells [78], suggesting that CD38 and CD157 are mutually exclusive in terms of expression, with the notable exception of the myeloid compartment.

The initial characterization of the structure and distribution of CD157 was performed by Todd et al., using the Mo-5 mAb [77]. Later on, a contribution to clustering as CD157 was independently provided by the cloning of BST-1, a molecule expressed by BM stromal cells and by similar lines derived from the stroma of rheumatoid arthritis patients [22]. The murine homologue of CD157 was cloned in the same period by Dong et al. [79].

CD157 shares the same enzymatic properties as CD38 and - as mentioned above - the two molecules have a similar genetic organization and a close chromosomal localization [23, 60, 80].

Again in analogy to CD38, CD157 acts as a receptor capable of generating signals which can be measured in terms of phosphorylation of a cascade of intracellular proteins. The signals mediated by CD157 were initially defined and characterized in the context of the interactions taking place between stromal and hemopoietic cells in closed environments, such as the BM or the joints $[22,81]$. The pathway induced upon CD157 cross-linking by a polyclonal antibody involved tyrosine phosphorylation of selected substrates in different line models [82-84], amply reviewed in [85]. These findings imply the existence of a cell bound ligand for CD157, potentially alternative to $\mathrm{NAD}^{+}$and $\mathrm{NADP}^{+}$, as was the case for CD38. The existence of a ligand is also supported by the finding that a soluble form of CD157 binds to human B-cell, lines [78].
However, the pathway controlled by CD157, the mechanisms of signal delivery, the molecular nature of the substrates involved and the identification of the ligand/counter-receptor await further investigation.

\section{Role of CD38 and CD157 in adhesion and migration}

A significant number of cell surface molecules involved in the processes of cell adhesion and migration have unexpectedly turned out to be enzymes [86]. A role for CD38 in cell adhesion was initially hypothesized more than a decade ago during analysis of the dynamic adhesion phenomena taking place between Tcell, if adjectival subsets and endothelial cells [87]. That work demonstrated involvement of CD38 in the early phases of lymphocyte adhesion to the endothelium [40] through direct interaction with CD31 [42]. CD38/CD31 interactions up-modulate integrin expression, thus promoting the ensuing phases in the adhesion cascade, and induce the synthesis and secretion of a panel of cytokines on $\mathrm{T}$ cells [88] and of prostaglandin on endothelial cells [89], suggesting the existence of a regulatory loop.

Another possibility is that these interactions depend on modulation of the enzymatic activities of CD38. This scenario seems likely in mice, where cADPR catalyzed by CD38 is essential for eliciting a sustained calcium response in murine neutrophils, triggering chemotactic movements to infected tissues [90]. The initial observation that $\mathrm{CD} 38 \mathrm{KO}$ mice are considerably more sensitive to intratracheal infections with culminated in the finding that the CD38 expressed by murine neutrophils is a modulator of innate immune responses to bacteria [91]. The effects mediated by CD38 are directly dependent on calcium mobilization regulated through cADPR production [91].

Transfer of these observations from mice to men has only been partial. For one thing, CD38 is expressed at minimal levels by normal human neutrophils, where, at least in resting conditions, the dominant enzyme is CD157. CD157 is a key molecule in the regulation of polarization and migration of neutrophils in humans, whereas the same has not been observed in mice. Further, the mechanisms differ significantly in that they are apparently independent of the enzymatic activity and rely mostly on localization in membrane rafts and on physical interaction with $\beta 2$ integrins [92].

A recent expansion of these findings to dendritic cells adds to the list of differences between men and mice. In both instances, CD38 is expressed by human and murine mature dendritic cells and in both instances it controls migration and polarization of $\mathrm{T}$ cells 


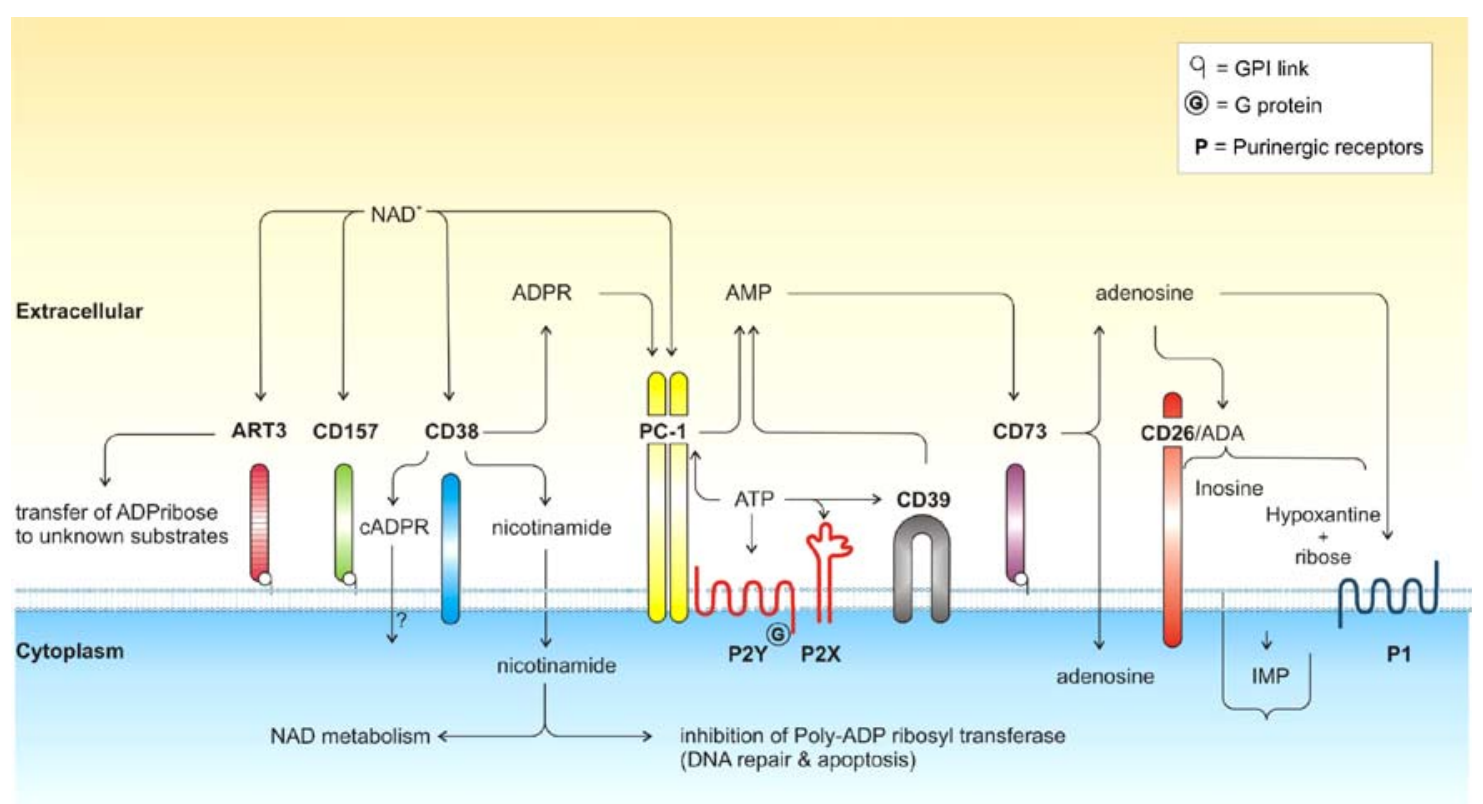

Fig. 1 Schematic representation of a virtual cell expressing a set of functionally related nucleotide-metabolizing cell surface enzymes. These enzymes are involved in (i) scavenging of nucleotides freed in the extracellular environment during inflammatory processes; (ii) modulation of purinergic receptor signaling and ART activity, and (iii) generation of immunomodulatory compounds, with extra- and intra-cellular effects.

towards a Th1 phenotype. However, in murine dendritic cells cADPR is the crucial second messenger regulating dendritic cell functions [93], whereas these events appear independent of cADPR production in humans $[49,94]$.

Researchers are still grappling with the challenge of devising a unifying view. One appealing possibility is that $\mathrm{CD} 38$ and CD157 provide a connection between calcium modulation via cADPR and classical signaling cascades mediated by the tyrosine phosphorylation of activating and inhibitory enzymes. The crystal structure of the molecules as well as biochemical evidence indicate that they may work as dimers or dimers of dimers [73, 95], creating a central cavity which could operate as a channel for the internalization of the enzymatic products. Following this hypothesis, the $\mathrm{NAD}^{+}$and $\mathrm{NADP}^{+}$present in the extracellular space would be trapped by CD38 and/or CD157 and the cADPR produced immediately 'microinjected' in the cytoplasm, eventually binding ryanodine receptors and inducing a calcium wave. This perturbation of calcium homeostasis might be responsible for the activation of calcium-dependent kinases, thus initiating a signaling cascade closely resembling a canonical receptorial pathway. CD31 and other putative ligands could act as modulators of the three-dimensional structure of CD38, modifying its propensity to bind the substrates and start a signaling event. The localization of CD38 and CD157 in critical areas of the plasma membrane in close association with signaling receptors would create the necessary coupling of enzymatic and receptorial machinery and would thus be a requisite for the signal to begin.

\section{CD38 and CD157 are part of a network of cell surface enzymes}

It seems that there is a natural blueprint linking CD38 and CD157 to other leukocyte ectoenzymes, which share signaling abilities, enzymatic functions and localization in specialized areas of the membrane. Clustering enzyme/receptor molecules may serve as a strategy for collecting precious elements from dead cells, pooling together signaling receptors and adhesion molecules, as well as generating products acting as recruiters of different cells. A schematic representation of a virtual cell shows the mutual advantages that encourage aggregation of the ectoenzymes (Fig. 1). For instance, $\mathrm{NAD}^{+}$and ATP, intracellular metabolites of energy metabolism, are candidates as signaling molecules in extracellular environments [96, 97]. It is hypothesized that high concentrations of $\mathrm{NAD}^{+}$and ATP would be present at sites of cell necrosis and tissue damage: When the compounds reach the extracellular compartment, their levels are controlled by a 


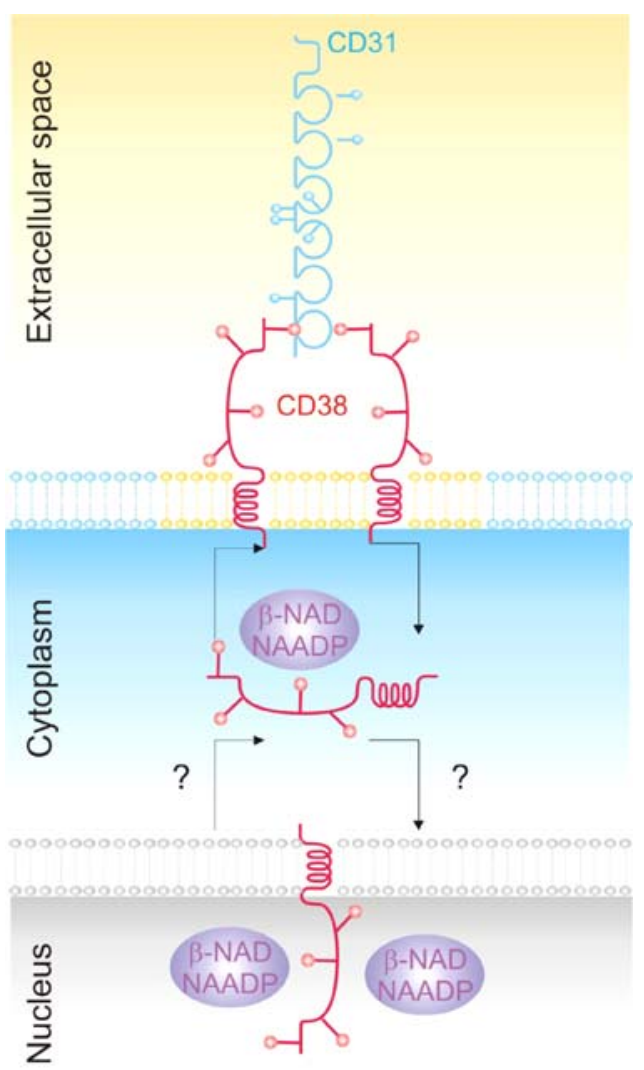

Fig. 2 Schematic model representing a possible dual function for CD38. Following this model, CD38 would perform as an adhesion/homing receptor when surface expressed and as an enzyme in the substrate-rich intracellular environments (cytoplasm and nucleus). The mechanisms exploited by CD38 to translocate to and from the three compartments are currently being analyzed.

number of ectoenzymes. This pathway has clearly been demonstrated for CD39 and related ectonucleotidases [98], which hydrolyze ATP to ADP and/or AMP. Similarly, Koch-Nolte et al. recently reported that CD38 enzymatically controls the availability of extracellular $\mathrm{NAD}^{+}$as a substrate for ADP-ribosyltransferase (ART)-catalyzed ADP-ribosylation of cell surface proteins [99].

\section{Conclusions}

The dual nature of CD38 can be formulated in terms of its independent functions as receptor and as enzyme, reflecting two separate strands in its long evolutionary history. A reasonable alternative, which finds itself the focus of intense but still unsuccessful investigation, is that $\mathrm{CD} 38$ is a receptor that exploits its enzymatic characteristics for signaling. These views reflect - or, more appropriately, are biased by - the points of view of the groups analyzing the molecules.
Immunologists tend to privilege the receptorial activities and the existence of a counter-receptor, fulfilling the needs for being a genuine adhesion/homing marker. Biochemists are attracted by the complexity of the functions exerted by these multifunctional and unique enzymes and their ability to regulate cytoplasmic calcium via an IP3-independent pathway.

Other controversies derive from the use of different models, mainly man and mouse. Most of the experiments on human samples have been performed on leukocytes derived from blood, while the evidences in murine and rat models are predominantly inferred from cells derived from lymph nodes and spleen. Nonetheless, we have also seen how the mouse system benefited from the existence of a CD38 KO model, enabling an accurate and causative comparison with the normal counterpart. The search for naturally occurring CD38- individuals was attempted in humans by analyzing more than 5,000 blood samples from newborns, reproducing the experience gathered with individuals lacking complement C2 and C4 [100]. In the Italian sample analyzed, none of the newborns was CD38-, suggesting that the absence of CD38 is incompatible with life (F. Malavasi, unpublished results). These results stand in apparent contradiction to the mouse model, where the $\mathrm{KO}$ animal is characterized by selected deficiencies in the immune effectors and innate responses, but it is still able to live and reproduce [90, 101]. The search for CD157 individuals (i.e., human $\mathrm{KO}$ ) was pursued in a sample of patients affected by paroxysmal nocturnal hemoglobinuria, a clonal disease characterized by the inability to synthesize the GPI linkage. Indeed, these patients are $\mathrm{CD} 157^{-}$in the pathological clone: However, this approach is not straightforward, for the inability to form GPI anchors is shared not only by CD157, but by all the molecules with this linkage to the membrane [92].

What does seem evident, however, is that we are faced with a family of molecules which descended from the same distant ancestors, but which diverged during evolution because of the pressures of natural selection and the different needs of the organisms. The cost of maintaining such systems must be counterbalanced by a definite profit for living systems. Another hint favoring a common view comes from analysis of the limited polymorphisms observed for the genes of human CD38 [23]. Indeed, evaluation of the allele distribution in a Caucasoid population reveals no differences between individuals from continental Italy and Sardinians, the latter considered the ancient core of the genetic reservoir of Europeans and whose unique characteristics have been conserved as a consequence of geographic restraints [102]. These 
findings suggest that the selective pressure behind the two alleles reaches far into the distant past. Future research would benefit from detailed study of the differences between homozygous and heterozygous individuals in terms of enzymatic functions and signal transduction ability. The relationship between CD38 and selected diseases may also indicate that the members of this ectoenzyme family played a role in the defense against (or, better, resistance to) the diseases that in more recent centuries modeled the genome that we see today. Perhaps the greatest challenge to resolving these questions and controversies rests in defining the proper framework within which to interpret the results achieved by the limited number of groups working on ectoenzymes.

Another possibility awaiting confirmation is that CD38 may play a double function, according to its localization (Fig. 2). Indeed, it may act as a receptor when surface expressed and as an enzyme when internalized upon request or during its cytoplasmic transit [103]. There is no current evidence that this model may also hold true for CD157, mainly because of its GPI anchor, nor has the role of soluble CD38 [104, 105] and CD157 [106] been analyzed in any great detail.

The CD38 paradigm is also representative of a more general pattern involving several nucleotide-metabolizing ectoenzymes, such as CD39 [98] and CD26 [107]. Indeed, it appears that all these enzymes evolved from very well conserved ancestors, usually acquiring a membrane anchorage (either transmembrane or GPI) and developing parallel and apparently independent functions. Relevant in an immunological context is the acquisition of functions classically associated with receptor activity, such as signal transduction. These include calcium mobilization and protein tyrosine phosphorylation [108]. Another common trait is localization in membrane lipid microdomains and physical and functional association with partners specialized in signal transduction $[109,110]$.

Acknowledgements Supported by grants from AIRC (Associazione Italiana Ricerca Cancro, Milano, Italy), FIRB (Fondo Investimenti Ricerca di Base, Rome, Italy), FIRMS (Fondazione Internazionale Ricerca Medicina Sperimentale,Torino, Italy), the Regione Piemonte and the University of Torino.

\section{References}

1. Goding JW, Howard MC (1998) Ecto-enzymes of lymphoid cells. Immunol Rev 161:5-10

2. De Flora A, Guida L, Franco L, Zocchi E (1997) The CD38/cyclic ADP-ribose system: a topological paradox. Int J Biochem Cell Biol 29:1149-1166
3. Deaglio S, Mehta K, Malavasi F (2001) Human CD38: a (r)evolutionary story of enzymes and receptors. Leuk Res 25:1-12

4. Malavasi F, Funaro A, Roggero S et al (1994) Human CD38: a glycoprotein in search of a function. Immunol Today 15:95-97

5. Kansas GS, Wood GS, Tedder TF (1991) Expression, distribution, and biochemistry of human CD39. Role in activation-associated homotypic adhesion of lymphocytes. J Immunol 146:2235-2244

6. Morimoto C, Schlossman SF (1998) The structure and function of CD26 in the T-cell immune response. Immunol Rev 161:55-70

7. Bene MC, Faure GC (1997) CD10 in acute leukemias. GEIL (Groupe d'Etude Immunologique des Leucemies). Haematologica 82:205-210

8. Goding JW, Terkeltaub R, Maurice M et al (1998) Ectophosphodiesterase/pyrophosphatase of lymphocytes and non-lymphoid cells: structure and function of the PC-1 family. Immunol Rev 161:11-26

9. Meuer SC, Acuto O, Hercend T, Schlossman SF, Reinherz EL (1984) The human T-cell receptor. Annu Rev Immunol 2:23-50

10. Terhorst C, van Agthoven A, LeClair K et al (1981) Biochemical studies of the human thymocyte cell-surface antigens T6, T9 and T10. Cell 23:771-780

11. Hercend T, Ritz J, Schlossman SF, Reinherz EL (1981) Comparative expression of T9, T10, and Ia antigens on activated human T cell subsets. Hum Immunol 3:247-259

12. Malavasi F, Funaro A, Alessio M et al (1992) CD38: a multi-lineage cell activation molecule with a split personality. Int J Clin Lab Res 22:73-80

13. Malavasi F, Caligaris-Cappio F, Milanese C et al (1984) Characterization of a murine monoclonal antibody specific for human early lymphohemopoietic cells. Hum Immunol 9:9-20

14. Katz F, Povey S, Parkar M et al (1983) Chromosome assignment of monoclonal antibody-defined determinants on human leukemic cells. Eur J Immunol 13:1008-1013

15. Funaro A, Spagnoli GC, Ausiello CM et al (1990) Involvement of the multilineage CD38 molecule in a unique pathway of cell activation and proliferation. $\mathrm{J}$ Immunol 145:2390-2396

16. Mehta K, Shahid U, Malavasi F (1996) Human CD38, a cell-surface protein with multiple functions. Faseb J 10:1408-1417

17. Lee HC (1998) Calcium signaling by cyclic ADP-ribose and NAADP. A decade of exploration. Cell Biochem Biophys 28:1-17

18. States DJ, Walseth TF, Lee HC (1992) Similarities in amino acid sequences of Aplysia ADP-ribosyl cyclase and human lymphocyte antigen CD38. Trends Biochem Sci 17:495

19. Howard M, Grimaldi JC, Bazan JF et al (1993) Formation and hydrolysis of cyclic ADP-ribose catalyzed by lymphocyte antigen CD38. Science 262:1056-1059

20. Zocchi E, Franco L, Guida L et al (1993) A single protein immunologically identified as CD38 displays NAD+ glycohydrolase, ADP-ribosyl cyclase and cyclic ADP-ribose hydrolase activities at the outer surface of human erythrocytes. Biochem Biophys Res Commun 196:1459-1465

21. Hirata Y, Kimura N, Sato K et al (1994) ADP ribosyl cyclase activity of a novel bone marrow stromal cell surface molecule, BST-1. FEBS Lett 356:244-248

22. Kaisho T, Ishikawa J, Oritani K et al (1994) BST-1, a surface molecule of bone marrow stromal cell lines that 
facilitates pre-B-cell growth. Proc Natl Acad Sci USA 91:5325-5329

23. Ferrero E, Saccucci F, Malavasi F (1999) The human CD38 gene: polymorphism, $\mathrm{CpG}$ island, and linkage to the CD157 (BST-1) gene. Immunogenetics 49:597-604

24. Kitanaka A, Ito C, Nishigaki H, Campana D (1996) CD38mediated growth suppression of B-cell progenitors requires activation of phosphatidylinositol 3-kinase and involves its association with the protein product of the c-cbl protooncogene. Blood 88:590-598

25. Kontani K, Kukimoto I, Nishina H et al (1996) Tyrosine phosphorylation of the c-cbl proto-oncogene product mediated by cell surface antigen CD38 in HL-60 cells. J Biol Chem 271:1534-1537

26. Mallone R, Funaro A, Zubiaur M et al (2001) Signaling through CD38 induces $\mathrm{NK}$ cell activation. Int Immunol 13:397-409

27. Zilber MT, Gregory S, Mallone R et al (2000) CD38 expressed on human monocytes: a coaccessory molecule in the superantigen-induced proliferation. Proc Natl Acad Sci USA 97:2840-2845

28. Zubiaur M, Izquierdo M, Terhorst C, Malavasi F, Sancho J (1997) CD38 ligation results in activation of the Raf-1/ mitogen-activated protein kinase and the CD3-zeta/zetaassociated protein-70 signaling pathways in Jurkat $T$ lymphocytes. J Immunol 159:193-205

29. Duan L, Reddi AL, Ghosh A, Dimri M, Band H (2004) The $\mathrm{Cbl}$ family and other ubiquitin ligases: destructive forces in control of antigen receptor signaling. Immunity 21:7-17

30. Deaglio S, Zubiaur M, Gregorini A et al (2002) Human CD38 and CD16 are functionally dependent and physically associated in natural killer cells. Blood 99:2490-2498

31. Deaglio S, Mallone R, Baj G et al (2001) Human CD38 and its ligand CD31 define a unique lamina propria $\mathrm{T}$ lymphocyte signaling pathway. Faseb J 15:580-582

32. Rah SY, Park KH, Han MK, Im MJ, Kim UH (2005) Activation of $\mathrm{CD} 38$ by interleukin- 8 signaling regulates intracellular $\mathrm{Ca} 2+$ level and motility of lymphokine-activated killer cells. J Biol Chem 280:2888-2895

33. Silvennoinen O, Nishigaki H, Kitanaka A et al (1996) CD38 signal transduction in human B cell precursors. Rapid induction of tyrosine phosphorylation, activation of syk tyrosine kinase, and phosphorylation of phospholipase Cgamma and phosphatidylinositol 3-kinase. J Immunol 156:100-107

34. Morra M, Zubiaur M, Terhorst C, Sancho J, Malavasi F (1998) CD38 is functionally dependent on the TCR/CD3 complex in human T cells. Faseb J 12:581-592

35. Moreno-Garcia ME, Lopez-Bojorques LN, Zentella A et al (2005) CD38 signaling regulates B lymphocyte activation via a phospholipase C (PLC)-gamma 2-independent, protein kinase $\mathrm{C}$, phosphatidylcholine-PLC, and phospholipase D-dependent signaling cascade. J Immunol 174:26872695

36. Munshi C, Aarhus R, Graeff R et al (2000) Identification of the enzymatic active site of CD38 by site-directed mutagenesis. J Biol Chem 275:21566-21571

37. Graeff R, Munshi C, Aarhus R, Johns M, Lee HC (2001) A single residue at the active site of CD38 determines its NAD cyclizing and hydrolyzing activities. J Biol Chem 276:12169-12173

38. Lund FE, Muller-Steffner HM, Yu N et al (1999) CD38 signaling in B lymphocytes is controlled by its ectodomain but occurs independently of enzymatically generated ADPribose or cyclic ADP-ribose. J Immunol 162:2693-2702
39. da Silva CP, Schweitzer K, Heyer P et al (1998) Ectocellular CD38-catalyzed synthesis and intracellular Ca2+signalling activity of cyclic ADP-ribose in T-lymphocytes are not functionally related. FEBS Lett 439:291-296

40. Dianzani U, Funaro A, DiFranco D et al (1994) Interaction between endothelium and CD4+CD45RA+ lymphocytes. Role of the human CD38 molecule. J Immunol 153:952-959

41. Deaglio S, Dianzani U, Horenstein AL et al (1996) Human CD38 ligand. A 120-KDA protein predominantly expressed on endothelial cells. J Immunol 156:727-734

42. Deaglio S, Morra M, Mallone R et al (1998) Human CD38 (ADP-ribosyl cyclase) is a counter-receptor of CD31, an Ig superfamily member. J Immunol 160:395-402

43. Deaglio S, Vaisitti T, Bergui L et al (2005) CD38 and CD100 lead a network of surface receptors relaying positive signals for B-CLL growth and survival. Blood 105:3042-3050

44. Wykes MN, Beattie L, Macpherson GG, Hart DN (2004) Dendritic cells and follicular dendritic cells express a novel ligand for CD38 which influences their maturation and antibody responses. Immunology 113:318-327

45. Cho YS, Han MK, Choi YB et al (2000) Direct interaction of the CD38 cytoplasmic tail and the Lck SH2 domain. $\mathrm{Cd} 38$ transduces $\mathrm{T}$ cell activation signals through associated Lck. J Biol Chem 275:1685-1690

46. Funaro A, De Monte LB, Dianzani U, Forni M, Malavasi F (1993) Human CD38 is associated to distinct molecules which mediate transmembrane signaling in different lineages. Eur J Immunol 23:2407-2411

47. Deaglio S, Capobianco A, Bergui L et al (2003) CD38 is a signaling molecule in B-cell chronic lymphocytic leukemia cells. Blood 102:2146-2155

48. Zilber MT, Setterblad N, Vasselon T et al (2005) MHC class II/CD38/CD9: a lipid-raft-dependent signaling complex in human monocytes. Blood 106:3074-3081

49. Frasca L, Fedele G, Deaglio S et al (2005) CD38 orchestrates migration, survival and Th1-immune responses of human mature dendritic cells. Blood 107:2392-2399

50. Tedoldi S, Paterson JC, Hansmann ML et al (2005) Transmembrane adaptor molecules: a new category of lymphoid cell markers. Blood 107:213-221

51. Zubiaur M, Fernandez O, Ferrero E et al (2002) CD38 is associated with lipid rafts and upon receptor stimulation leads to Akt/protein kinase B and Erk activation in the absence of the CD3-zeta immune receptor tyrosine-based activation motifs. J Biol Chem 277:13-22

52. Munoz P, Navarro MD, Pavon EJ et al (2003) CD38 signaling in $\mathrm{T}$ cells is initiated within a subset of membrane rafts containing Lck and the CD3-zeta subunit of the T cell antigen receptor. J Biol Chem 278:50791-50802

53. Lund FE, Yu N, Kim KM, Reth M, Howard MC (1996) Signaling through CD38 augments B cell antigen receptor (BCR) responses and is dependent on BCR expression. $\mathrm{J}$ Immunol 157:1455-1467

54. Ausiello CM, Urbani F, la Sala A, Funaro A, Malavasi F (1995) CD38 ligation induces discrete cytokine mRNA expression in human cultured lymphocytes. Eur J Immunol 25:1477-1480

55. Ausiello CM, la Sala A, Ramoni C et al (1996) Secretion of IFN-gamma, IL-6, granulocyte-macrophage colony-stimulating factor and IL-10 cytokines after activation of human purified T lymphocytes upon CD38 ligation. Cell Immunol 173:192-197

56. Damle RN, Wasil T, Fais F et al (1999) Ig V gene mutation status and CD38 expression as novel prognostic indicators in chronic lymphocytic leukemia. Blood 94:1840-1847 
57. Morabito F, Mangiola M, Oliva B et al (2001) Peripheral blood CD38 expression predicts survival in B-cell chronic lymphocytic leukemia. Leuk Res 25:927-932

58. Morabito F, Mangiola M, Stelitano C et al (2002) Peripheral blood CD38 expression predicts time to progression in B-cell chronic lymphocytic leukemia after first-line therapy with high-dose chlorambucil. Haematologica 87:217-218

59. Aarhus R, Graeff RM, Dickey DM, Walseth TF, Lee HC (1995) ADP-ribosyl cyclase and CD38 catalyze the synthesis of a calcium-mobilizing metabolite from NADP. J Biol Chem 270:30327-30333

60. Schuber F, Lund FE (2004) Structure and enzymology of ADP-ribosyl cyclases: Conserved enzymes that produce multiple calcium mobilizing metabolites. Curr Mol Med 4:249-261

61. Ceni C, Muller-Steffner H, Lund F et al (2003) Evidence for an intracellular ADP-ribosyl cyclase/NAD+-glycohydrolase in brain from CD38-deficient mice. J Biol Chem 278:40670-40678

62. Lee HC (2001) Physiological functions of cyclic ADPribose and NAADP as calcium messengers. Annu Rev Pharmacol Toxicol 41:317-345

63. Franco L, Zocchi E, Usai C et al (2001) Paracrine roles of $\mathrm{NAD}+$ and cyclic ADP-ribose in increasing intracellular calcium and enhancing cell proliferation of $3 \mathrm{~T} 3$ fibroblasts. J Biol Chem 276:21642-21648

64. Chini EN, Chini CC, Barata da Silva H, Zielinska W (2002) The cyclic-ADP-ribose signaling pathway in human myometrium. Arch Biochem Biophys 407:152-159

65. Deshpande DA, Dogan S, Walseth TF et al (2004) Modulation of calcium signaling by interleukin-13 in human airway smooth muscle: role of CD38/cyclic adenosine diphosphate ribose pathway. Am J Respir Cell Mol Biol 31:36-42

66. Podesta M, Pitto A, Figari O et al (2003) Cyclic ADP-ribose generation by CD38 improves human hemopoietic stem cell engraftment into NOD/SCID mice. Faseb J 17:310-312

67. Takasawa S, Nata K, Yonekura H, Okamoto H (1993) Cyclic ADP-ribose in insulin secretion from pancreatic beta cells. Science 259:370-373

68. Chini EN, Chini CC, Kato I, Takasawa S, Okamoto H (2002) CD38 is the major enzyme responsible for synthesis of nicotinic acid-adenine dinucleotide phosphate in mammalian tissues. Biochem J 362:125-130

69. Perraud AL, Fleig A, Dunn CA et al (2001) ADP-ribose gating of the calcium-permeable LTRPC2 channel revealed by Nudix motif homology. Nature 411:595-599

70. Basile G, Taglialatela-Scafati O, Damonte G et al (2005) ADP-ribosyl cyclases generate two unusual adenine homodinucleotides with cytotoxic activity on mammalian cells. Proc Natl Acad Sci USA 102:14509-14514

71. Ferrero E, Saccucci F, Malavasi F (2000) The making of a leukocyte receptor: origin, genes and regulation of human CD38 and related molecules. Chem Immunol 75:1-19

72. Nata K, Takamura T, Karasawa T et al (1997) Human gene encoding CD38 (ADP-ribosyl cyclase/cyclic ADP-ribose hydrolase): organization, nucleotide sequence and alternative splicing. Gene 186:285-292

73. Liu Q, Kriksunov IA, Graeff R et al (2005) Crystal structure of human CD38 extracellular domain. Structure (Camb) 13:1331-1339

74. Prasad GS, McRee DE, Stura EA et al (1996) Crystal structure of Aplysia ADP ribosyl cyclase, a homologue of the bifunctional ectozyme CD38. Nat Struct Biol 3:957-964
75. Love ML, Szebenyi DM, Kriksunov IA et al (2004) ADPribosyl cyclase; crystal structures reveal a covalent intermediate. Structure (Camb) 12:477-486

76. Yamamoto-Katayama S, Ariyoshi M, Ishihara $\mathrm{K}$ et al (2002) Crystallographic studies on human BST-1/CD157 with ADP-ribosyl cyclase and NAD glycohydrolase activities. J Mol Biol 316:711-723

77. Todd RF III, Roach JA, Arnaout MA (1985) The modulated expression of Mo5, a human myelomonocytic plasma membrane antigen. Blood 65:964-973

78. Ishihara K, Hirano $\mathrm{T}$ (2000) BST-1/CD157 regulates the humoral immune responses in vivo. Chem Immunol 75:235255

79. Dong C, Wang J, Neame P, Cooper MD (1994) The murine BP-3 gene encodes a relative of the CD38/NAD glycohydrolase family. Int Immunol 6:1353-1360

80. Hussain AM, Lee HC, Chang CF (1998) Functional expression of secreted mouse BST-1 in yeast. Protein Expr Purif 12:133-137

81. Shimaoka Y, Attrep JF, Hirano T et al (1998) Nurse-like cells from bone marrow and synovium of patients with rheumatoid arthritis promote survival and enhance function of human B cells. J Clin Invest 102:606-618

82. Okuyama Y, Ishihara K, Kimura N et al (1996) Human BST-1 expressed on myeloid cells functions as a receptor molecule. Biochem Biophys Res Commun 228:838-845

83. Liang F, Qi RZ, Chang CF (2001) Signalling of GPIanchored CD157 via focal adhesion kinase in MCA102 fibroblasts. FEBS Lett 506:207-210

84. Liang F, Qi RZ, Chang CF (2002) CD157 undergoes ligandindependent dimerization and colocalizes with caveolin in CHO and MCA102 fibroblasts. Cell Signal 14:933-939

85. Ortolan E, Vacca P, Capobianco A et al (2002) CD157, the Janus of CD38 but with a unique personality. Cell Biochem Funct 20:309-322

86. Salmi M, Jalkanen S (2005) Cell-surface enzymes in control of leukocyte trafficking. Nat Rev Immunol 5:760-771

87. Dianzani U, Malavasi F (1995) Lymphocyte adhesion to endothelium. Crit Rev Immunol 15:167-200

88. Deaglio S, Mallone R, Baj G et al (2000) CD38/CD31, a receptor/ligand system ruling adhesion and signaling in human leukocytes. Chem Immunol 75:99-120

89. Gurubhagavatula I, Amrani Y, Pratico D et al (1998) Engagement of human PECAM-1 (CD31) on human endothelial cells increases intracellular calcium ion concentration and stimulates prostacyclin release. J Clin Invest 101:212-222

90. Partida-Sanchez S, Randall TD, Lund FE (2003) Innate immunity is regulated by CD38, an ecto-enzyme with ADPribosyl cyclase activity. Microbes Infect 5:49-58

91. Partida-Sanchez S, Cockayne DA, Monard S et al (2001) Cyclic ADP-ribose production by CD38 regulates intracellular calcium release, extracellular calcium influx and chemotaxis in neutrophils and is required for bacterial clearance in vivo. Nat Med 7:1209-1216

92. Funaro A, Ortolan E, Ferranti B et al (2004) CD157 is an important mediator of neutrophil adhesion and migration. Blood 104:4269-4278

93. Partida-Sanchez S, Goodrich S, Kusser K et al (2004) Regulation of dendritic cell trafficking by the ADP-ribosyl cyclase CD38: impact on the development of humoral immunity. Immunity 20:279-291

94. Fedele G, Frasca L, Palazzo R et al (2004) CD38 is expressed on human mature monocyte-derived dendritic 
cells and is functionally involved in CD83 expression and IL-12 induction. Eur J Immunol 34:1342-1350

95. Moreno-Garcia ME, Partida-Sanchez S, Primack J et al (2004) CD38 is expressed as noncovalently associated homodimers on the surface of murine B lymphocytes. Eur J Biochem 271:1025-1034

96. Ziegler M (2000) New functions of a long-known molecule. Emerging roles of NAD in cellular signaling. Eur J Biochem 267:1550-1564

97. la Sala A, Ferrari D, Di Virgilio F et al (2003) Alerting and tuning the immune response by extracellular nucleotides. J Leukoc Biol 73:339-343

98. Robson SC, Wu Y, Sun X et al (2005) Ectonucleotidases of CD39 family modulate vascular inflammation and thrombosis in transplantation. Semin Thromb Hemost 31:217-233

99. Krebs C, Adriouch S, Braasch F et al (2005) CD38 controls ADP-ribosyltransferase-2-catalyzed ADP-ribosylation of T cell surface proteins. J Immunol 174:3298-3305

100. Malavasi F, Olivetti E, Milanese C, Carbonara AO (1981) Properdin factor B polymorphism in continental Italy and Sardinia. Hum Genet 58:209-212

101. Cockayne DA, Muchamuel T, Grimaldi JC et al (1998) Mice deficient for the ecto-nicotinamide adenine dinucleotide glycohydrolase CD38 exhibit altered humoral immune responses. Blood 92:1324-1333

102. Piazza A (1999) DNA polymorphisms of genetic diseases in Italy. J Biol Regul Homeost Agents 13:1-7
103. Deaglio S, Malavasi F (2002) Human CD38: a receptor, an (ecto)enzyme, a disease marker and lots more. Modern Aspct Immunobiol 2:121-125

104. Funaro A, Horenstein AL, Calosso L et al (1996) Identification and characterization of an active soluble form of human CD38 in normal and pathological fluids. Int Immunol 8:1643-1650

105. Lebedev MJ, Egorova NI, Sholkina MN et al (2004) Serum levels of different forms of soluble CD38 antigen in burned patients. Burns 30:552-556

106. Sato A, Yamamoto S, Kajimura N et al (1999) Inhibitor peptide SNP-1 binds to a soluble form of BST-1/CD157 at a 2:2 stoichiometry. Eur J Biochem 264:439-445

107. De Meester I, Korom S, Van Damme J, Scharpe S (1999) CD26, let it cut or cut it down. Immunol Today 20:367-375

108. Ishii T, Ohnuma K, Murakami A et al (2001) CD26mediated signaling for $\mathrm{T}$ cell activation occurs in lipid rafts through its association with CD45RO. Proc Natl Acad Sci USA 98:12138-12143

109. Kittel A, Csapo ZS, Csizmadia E, Jackson SW, Robson SC (2004) Co-localization of P2Y1 receptor and NTPDase1/ CD39 within caveolae in human placenta. Eur J Histochem 48:253-259

110. Pacheco R, Martinez-Navio JM, Lejeune M et al (2005) CD26, adenosine deaminase, and adenosine receptors mediate costimulatory signals in the immunological synapse. Proc Natl Acad Sci USA 102:9583-9588 